\title{
Self-study en el diseño de la estrategia metodológica de Aprendizaje Basado en un Proyecto Docente (ABPD) para la formación de profesorado
}

\section{Self-study in the design of the methodological strategy of Learning Based on a Teaching Project for teacher training}

\author{
$\mathrm{M}^{\mathrm{a}}$ Covadonga de la Iglesia Villasol (1) @ \\ Universidad Complutense de Madrid (UCM), España
}

\begin{abstract}
Resumen
Tras más de una década de formación a futuros docentes, la autoreflexión sobre la práctica para el aprendizaje es inevitable, para interpretar, construir y definir el saber hacer en la profesión, propia y de los futuros docentes. En la formación de éstos, la creatividad es condición necesaria para servir de transmisor del saber hacer y favorece el acercamiento multidisciplinar en el aprendizaje, donde las TIC, en la era digital, sirven de hilo conductor en la coordinación de recursos, contenidos, y estrategias didácticas. Este entorno virtual se muestra excepcionalmente útil para aportar una visión radial e integral de los procesos de enseñanza y aprendizaje, para quienes cursan el Máster de Formación del Profesorado (MFP), que potencia el desarrollo de competencias y/o habilidades requeridas en el entorno profesional al que se enfrentan, la enseñanza y transmisión de conocimientos. En este trabajo se muestra, a modo de caso, el proceso reflexivo personal seguido en la implantación de una propuesta integral de Aprendizaje Basado en un Proyecto Docente (ABPD), que se ha gestado desde la puesta en marcha del MFP en la UCM en la asignatura de Didáctica en la especialidad de Economía y Administración de Empresas (ADE y ECO), desde el enfoque del self-study en la formación práctica y pedagógica, conjugando el binomio universidad y centros educativos de secundaria.
\end{abstract}

Palabras clave: auto-estudio; creatividad; digital; multidisciplinar; aprendizaje; habilidades.

\begin{abstract}
After more than a decade of training for future teachers, self-reflection on the practice for learning is inevitable, to interpret, build and define the know-how in the profession, own and of future teachers. In the formation of these, creativity is a necessary condition to serve as a transmitter of know-how and favors the multidisciplinary approach in learning, where ICTS, in the digital era, serve as a guiding thread in the coordination of resources, contents, and didactic strategies. This virtual environment is exceptionally useful to provide a radial and integral vision of the teaching and learning processes, for those who take the Master's Degree in Teacher Training (MFP), which promotes the development of skills and / or skills required in the professional environment they face, the teaching and transmission of knowledge. This work shows, as a study case, the personal reflective process developed in the implementation of a comprehensive proposal for Learning Based on a Teaching Project, which has been developed since the becoming of the MFP at the UCM in the Didactics subject in the specialty of Economics and Business Administration (ADE and ECO), from the focus of self-study in practical and pedagogical training, combining the university and secondary education centers.
\end{abstract}

Keywords: self-study; creativity; digital; multidisciplinary; learning; skills.

Recomendación de cómo citar este artículo (APA 6)

Iglesia, M. C. de la (2020). Self-study en el diseño de la estrategia metodológica de Aprendizaje Basado en un Proyecto Docente (ABPD) para la formación de profesorado. Revista Iberoamericana de Educación, 82(2),81-106. https://doi.org/10.35362/rie8223526 


\section{Introducción, justificación y motivación del self-study}

Tres elementos constituyen el núcleo de este trabajo. En primer lugar, la necesaria reflexión fruto del ejercicio de la profesión docente en interacción con profesionales que quieren ser futuros profesores, en el área de Economía y Administración de Empresas (ECO y ADE) del Máster de Formación del Profesorado (MFP) 1,2. Esta docencia ha motivado una continua redefinición de los roles a desempeñar, y que principalmente implican convertirse en un co-aprendiz de su profesión de docente (Iglesia, 2012) y un orquestador, con funciones administrativas, pedagógicas, tecnológicas y de enlace (Wake, Dysthe y Mjelstad, 2007), así como un generador de un nuevo conocimiento y rediseño del currículo del futuro docente (Terigi, 2012), un consultor de información, colaborador en grupo, facilitador, proveedor de recursos y supervisor académico (Gilbert, 2001). En segundo lugar, reconocer la importancia de fomentar la creatividad como el hilo conductor de la experiencia que se aporta en este trabajo, motor de la innovación y factor clave para intensificar el desarrollo de las competencias personales, laborales, empresariales y sociales de los individuos, así como el bienestar social³. Y, en tercer lugar, la rápida incorporación de las TIC al entorno cultural, social y educativo, que bidireccionalmente permite la creación, intercambio y distribución masiva de contenidos de entretenimiento, educación o comunicación, en diversos formatos, televisivo, radiofónico, impresión, internet, canales multimedia, videos, etc., y tanto entre la población joven, que ya son nativos digitales, como por los usuarios de mayor edad (Pascu, 2008).

Ahondando en esta transformación, según Iglesia (2018), el profesor deja de ser exclusivamente un mero experto en contenidos docentes, diseñador de nuevos entornos de aprendizaje en combinación de diversos recursos y técnicas educativas, a la vez que un generador y evaluador de recursos útiles para el autoaprendizaje, sin olvidar la faceta de ser orientador, tutor y motivador, con el triple objetivo de 1) transmitir conocimiento técnico y especializado, 2) favorecer que los estudiantes y futuros docentes desarrollen las competencias requeridas en el entorno profesional,

\footnotetext{
${ }^{1}$ Master obligatorio y profesionalizante para los futuros docentes en centros de educación secundaria y de formación profesional.

2 Se agradece a los alumnos su generosidad y disponibilidad para permitir el uso público de algunos materiales elaborados y rescatados del blog de acceso público.

${ }^{3}$ La declaración de 2009 como el Año Europeo de la Creatividad y la Innovación, con el objetivo de respaldar los esfuerzos de las instituciones para promover la creatividad por medio del aprendizaje permanente, ha sido detonante de numerosos estudios. Decisión Nº 1350/2008/EC OF, European Parliament and of the Council, of 16, december 2008, Official Journal of the European Union.
} 
y 3) ser un estudioso del proceso pedagógico observado en la práctica (self-study), propia y de otros, convirtiéndose para sus estudiantes y futuros docentes en un acompañante en el proceso formativo.

Cuando el docente lo es de futuros profesores en formación, asume, además, el rol de observador del aprendizaje de sus estudiantes, en un periodo de entrenamiento o simulación de la profesión docente. En dicho periodo, la gestión y el análisis de la información extraída (analista de datos) referida por Harrison y Killion (2007), permite al docente conocer mejor su propia acción docente y tutorial, la tipología de sus estudiantes, sus resultados, sus actitudes y su compromiso con la profesión. Así, se requiere de una continua y necesaria observación pedagógica de la interacción entre la universidad y el centro educativo al que se integrarán los estudiantes en su periodo de formación (Russell, Fuentealba e Hirmas, 2016), los cuales ejercerán como docentes en una sociedad, presente y futura, caracterizada por su flexibilidad, volatilidad y liquidez, cuyo avance continuo genera cambios en la forma de aprender, organizarse, comunicarse, gestionar la información y relacionarse (Echevarría y Martínez, 2018).

En este contexto, las instituciones educativas han adoptado con entusiasmo y celeridad las TIC, tanto en las actividades docentes curriculares como en aquellas que realizan para acercarse al ciudadano, transferir el conocimiento y la investigación, ahondar en la sensibilización, y cómo no, fomentar la profesionalización docente, empoderando a los formadores de los futuros docentes (en todas las acepciones de la RAE: "dar a alguien autoridad, influencia o conocimiento para hacer algo").

Estas tecnologías ${ }^{4}$ diseñan nuevos, versátiles e imprevisibles espacios de enseñanzaaprendizaje, formas de relacionarse, de búsqueda y procesamiento de la ingente cantidad de información accesible, nuevas normas de conducta y, en definitiva, nuevos modelos, a los que el docente debe ir readaptando su actividad docente, para lo cual en muchos casos debe, a su vez, adquirir, desarrollar o reforzar una serie de capacidades genéricas y transversales. El ya superado debate sobre la oportunidad de utilizar las TIC en el aula ha dejado sitio a un doble reto: i) cómo utilizarlas coherentemente, como instrumento que aporte conocimiento, transmisor

\footnotetext{
${ }^{4}$ Las TIC han modificado el diseño, gestión y desarrollo de los proyectos de e-Aprendizaje, de enseñanza abierta y a distancia (EAD), generando diversos modelos: autoaprendizaje tutorizado, el aula virtual sincrónica y el aprendizaje cooperativo en red, con características pedagógicas, tecnologías, modos de implantación, contenido formativo y las habilidades que debe tener o desarrollar el estudiante, distintas según modelo (Dondi, 2001).
} 
de valores y desarrollador de competencias profesionales, y ii) cómo evaluar su uso en un entorno digital sumamente cambiante, en el que, en general, los estudiantes (ya nativos digitales) son más hábiles o expertos en su uso.

En este contexto educativo se muestra cada vez más necesaria la reflexión y valoración crítica en la acción docente y sobre la acción docente, self-study, (Schön, 1988), para darle significado, interpretar y construir el saber profesional del formador. $Y$ esto bajo un criterio evolutivo y de continuo reajuste, construyendo conocimiento a partir de la práctica docente, estudiando nuestro propio trabajo docente, y aceptando tal enseñanza como una disciplina y por tanto como posible objeto de investigación (Loughran y Russell, 2016), de naturaleza colaborativa, enfocada a la mejora e interactividad (LaBoskey, 2004), y en general a partir de métodos cualitativos y relatos documentales.

Así, en el caso que se presenta, ha resultado determinante incorporar como faro orientador el fomento de la creatividad, no solo puntualmente, sino de forma continua e irradiada a lo largo de la vida y en todos los aspectos, apostando por proveer un ambiente, de estudio y/o trabajo, que estimule la innovación, la flexibilidad y la adaptabilidad a los cambios que, de forma tan rápida y en modos tan diversos, se están desarrollando en el entorno educativo y profesional, favoreciendo la transmisión bidireccional de la creatividad a todos los contextos, desde el aprendizaje, el desarrollo profesional, la sensibilidad estética, el desarrollo emocional, la intuición o el pensamiento lateral (Iglesia, 2012).

Por tanto, este trabajo reflexiona, desde la metodología del self-study ${ }^{5}$, sobre las lecciones aprendidas antes y durante el proceso de reformulación de la propuesta metodológica integral de Aprendizaje Basado en un Proyecto Docente (ABPD), que gira en torno a tres elementos creatividad, digitalidad y aprender la profesión, tanto para el propio docente como los estudiantes y futuros profesores (Iglesia, 2018). Este ejercicio de self-study muestra la evolución reflexiva personal en contacto con futuros docentes que se encuentran en otras etapas vitales menos maduras, con otras experiencias profesionales y expectativas de futuro, y con otras percepciones sobre la profesión docente, como también apuntan Conde-Jiménez y Martín-Gutiérrez (2016). Ha sido un viaje reflexivo al interior del aula como un espacio activo de enseñanza, parándonos a pensar, reflexionar y actuar, tratando de responder a preguntas clave sobre cómo se aprende a enseñar y cómo se enseña a enseñar (Cabrera-Murcia, Silva y Gorichón, 2020).

${ }^{5}$ El autor ha conocido esta disciplina principalmente a través de Samaras (2002). Un trabajo orientador y motivador ha sido Russell, Hirmas y Fuentealba (2016). 


\section{Contexto, Objetivos y Metodología del ejercicio de self-study}

El desarrollo del ejercicio de self-study se circunscribe al entorno de la implantación de la propuesta metodológica integral de Aprendizaje Basado en un Proyecto Docente (ABPD), en el bloque de Didáctica en el Máster de Formación de Profesorado (MFP), en la especialidad de Economía y Administración de Empresas, en la UCM. Recoge, pues, la autoreflexión en combinación con aspectos reflexivos comunes con los estudiantes y futuros docentes, tratando de dar respuesta a las demandas y desafíos continuos a los que se enfrentarán en su ejercicio profesional. Este ejercicio exige conectar la propia realidad como docente a la realidad del entorno educativo y de mis propios alumnos como futuros docentes, extrayendo así un feedback que reformule la identidad como formador de formadores.

Según las premisas previas, los objetivos de este ejercicio son:

1. Estructurar, explorar y analizar la propia experiencia como formador de futuros docentes.

2. Extraer elementos de mejora, tanto para mi propia acción docente como de los docentes en formación.

En cuanto a la metodología, el ejercicio de self-study se construye sobre una serie de elementos que responden, con alguna particularidad, a la estructura diseñada por Johns (2010): i) la propia observación reflexiva, ii) la revisión de notas personales a modo de diario y iii) la reflexión compartida con los acompañantes (amigos) críticos, en los términos que expresan Rusell, Fuentealba e Hirmas (2016) y Rusell (2017). En concreto, las fases seguidas son:

1. Reflexión sobre la experiencia docente pasada.

2. Autoreflexión valorativa.

3. Reflexión sobre lo que ocurre en el entorno.

4. Reflexión sobre la base documental.

5. Reflexión compartida con los docentes en formación.

6. Reflexión en clave de acciones de mejora y consolidación.

El estudio de caso self-study, ha sido desarrollado sobre diversos métodos interpretativos, para recoger información variada procedente de múltiples fuentes, interconectada entre sí, combinando fuentes cuantitativas (cuestionarios) con pequeños apuntes o ensayos reflexivos, valorativos, breves entrevistas, en diversos momentos del curso, tanto para el docente como los estudiantes y futuros docentes: 1) el cuestionario sobre atributos o cualidades del docente y el cuestionario sobre 
el grado de desarrollo competencial e importancia, administrado al inicio y final del curso con aporte reflexivo. 2) Recogida de notas valorativas puntuales sobre ciertas actividades, recursos o dinámicas, que constituyen las denominadas "catas de último minuto". 3) Ensayos de valoración de las actividades o recursos presentados por un compañero, como en la evaluación por pares, comparado con el suyo propio. 4) Notas de campo de las prácticas y exposiciones, seminarios o reuniones de trabajo realizadas. 5) Un diario de actividad del docente, con indicaciones reflexivas. Y, 6) Colección y catalogación de recursos docentes elaborados por los estudiantes en su práctica que configuran el portafolio que presentan en público.

En cuanto a los cuestionarios de valoración diseñados ad-hoc, han permitido un análisis descriptivo cuantitativo, utilizado en algunos casos para ajustar y concretar algunos elementos reflexivos y en otros como el detonante para la reflexión comunitaria, y que en general no se presentan por exceder el contexto de esta investigación.

Por tanto, tras el diseño expuesto, el ejercicio de self-study se estructura en tres apartados, que responden a tres momentos claves del proceso de implantación de la estrategia ABPD y autorreflexión valorativa: los antecedentes como desencadenantes de la dinámica, la simultánea a la implantación y la posterior reflexión que aportará un feedback propio y externo como elementos de mejora continua. Obviamente el ejercicio relaciona dimensiones como: motivación, actitudes, competencias, emociones, miedos, apoyo externo, valoración crítica y constructiva, fortalezas y debilidades y acciones de mejora.

\section{Desarrollo del ejercicio de self-study}

\subsection{Antecedentes e identificación de una estrategia diferenciada: pre self-study del ABPD}

Un elemento que ha sido el detonante de un cambio cualitativo en la reflexión de la propia acción docente se fecha en 2010, iniciando un proceso de rediseño consciente de los principios pedagógicos a aplicar en una enseñanza orientada al aprender-haciendo. En concreto, estando inmersa en la revisión exhaustiva de algunos trabajos de investigación para un artículo sobre las aportaciones de las primeras mujeres economistas a la Ciencia Económica, un grupo de estudiantes de la especialidad de Análisis Económico conocedores de esa aventura, recuperaron y me regalaron el texto Ensayos sobre Análisis Económico, de Robinson (1959, reimpreso en 1974). Me adentré, sin pretenderlo, en una lectura apasionada de 
aspectos metodológicos, principalmente del tan acertado concepto, la caja de herramientas (en el original a box of tools, y que según el contexto podría ser traducido como caja de habilidades), acuñado por la profesora Robinson ${ }^{6}$ para describir sin ambigüedad los elementos básicos que permiten formalizar el estudio de los principios económicos. La plasticidad de este concepto siempre me ha parecido imprescindible para identificar las herramientas metodológicas que el docente debe incorporar en el proceso de enseñanza, sea cual sea el área de conocimiento, y que hacen de la educación un proceso integrador y transversalizado, perfectamente adaptable a cualquier profesión, al evidenciar la necesidad de identificar las herramientas (criterios metodológicos, recursos, prácticas docentes, usos didácticos, elementos y aplicaciones digitales, etc.), que perfilan una actividad profesional, en este caso la del docente formador de futuros profesores.

Dicho detonante creó un interés nuevo tanto por el estudio de los planteamientos metodológicos, recuperando algunos elementos de la faceta docente tanto de Robinson como de otros ilustres académicos, perfectamente vigentes en el contexto educativo actual, como por la reflexión valorativa sobre el quehacer docente, por cuanto que la formación de profesores reflexivos es ya una corriente de estudio e investigación muy fructífera, en el entorno educativo anglosajón desde finales de los 1990, extendiéndose a otros países y sistemas educativos (Zeichner, 2010).

Así, algunos párrafos del prólogo del texto Ensayos sobre Análisis Económico, identifican elementos metodológicos (herramientas), para que el estudiante,... aprenda algo haciéndolo,...pueda continuar por sí mismo a partir del punto donde termina el libro, y...use (los ejercicios) como base de una discusión en grupo, refiriéndose, además, al método de pensamiento y de estudio como ... un modelo de construcción, que...consiste en un cuadro mental muy simplificado, que exhibe el comportamiento de la gente en un medio social y físico, y que elimina lo que no es esencial... Pues bien, éstas píldoras metodológicas han acompañado y reorientado la reflexión sobre cómo abordar la acción docente en su transposición a la era digital sobre la que transitamos, siendo la tesis a desarrollar que las herramientas docentes deben ser ambivalentes, integradoras, no exclusivistas, multidimensionales y multiformato. 6 "...In Mrs. Robinson's unsurpassably felicitous phrase, economic theory is a box of tools".
Schumpeter (1954, p.15). 
Se considera, por tanto, que todo docente debe reconocer qué elementos de su caja de herramientas han dejado de ser útiles en su ejercicio docente, cuáles, siendo nuevas, puede ser incorporadas exitosamente, y cuáles, dadas sus propias destrezas, conociéndolas, no se ajustan a su estilo y práctica, siempre a partir de la autoevaluación y reflexión de su práctica docente pasada y presente.

Adicionalmente, cuatro hechos han contribuido a identificar la necesidad de plantear, estructurar e implementar la estrategia integradora de Aprendizaje Basado en un Proyecto Docente (ABPD), como son:

1. La trayectoria, durante más de quince años, de participar en equipos innovadores multidisciplinares en la propia universidad, desarrollando diversos proyectos de innovación y mejora de la calidad docente, adentrándose en el uso de nuevas herramientas digitales, estrategias docentes, metodologías activas, desarrollo de competencias, etc., y que han supuesto a su vez una fuente de experimentación e investigación sobre la propia acción docente.

2. La necesidad de adquirir una formación complementaria sobre metodologías didácticas y roles pedagógicos, así como transitar por áreas de investigación referidas a temas educativos y docentes, como son el absentismo, la incorporación de las TIC, el estudio y valoración de los atributos docentes, potenciar la creatividad en el aula o la medición del grado de desarrollo de competencias según opinión de los estudiantes, etc.;

3. La autorreflexión en el proceso de adaptación de la Educación Superior Española a los criterios de Bolonia7, que dibujó un nuevo mapa de titulaciones, redefiniendo el cómputo de créditos ECTS ${ }^{8}$, identificando tanto el esfuerzo del profesor como del estudiante de una docencia volcada en el alumno, y creó la necesidad de reinventar los procesos de enseñanza aprendizaje sobre el principio "aprender-haciendo" en la transmisión de conocimientos y el desarrollo de las habilidades transversales y socio-personales que demanda el entorno laboral.

4. Hacerse cargo de una parte de la docencia del bloque temático de la materia de didáctica en la especialidad de ECO y ADE del MFP, lo que exigió una revisión de materiales y contenidos a trabajar según el diseño del plan de estudios, la colaboración con otros docentes de otras áreas, la coordinación de actividades entre los distintos docentes, el análisis de las prácticas docentes en los colegios y centros educativos en los que los estudiantes realizarían el prácticum, así como supervisar y autorizar los aspectos docentes del mismo, y dirigir y evaluar tesinas o trabajos final de máster (TFM), en disciplinas muy distintas a la de la propia especialización, que es el análisis económico y la economía industrial.

\footnotetext{
${ }^{7}$ Declaración de Bolonia de 1999, ratificada por 32 países en el comunicado de Praga de 2001, del proceso de convergencia en el Espacio Europeo de Educación Superior.

${ }^{8}$ European Credit Transfer System.
} 
Estos anclajes se vieron reforzados con dos pilares:

a) La incorporación paulatina de diversas herramientas digitales o TIC a la docencia, que aportaron ventajas, ya señaladas por Carrasco, Gracia e Iglesia (2004), como ahondar en la formación centrada en el estudiante, adaptable según características y necesidades, flexible, superadora de barreras espacio-temporales, generadora de contenidos cuasi-inmediatos y económicos, que extiende la formación masivamente, aumenta la capacidad de interacción y comunicación, y que diseña nuevos espacios de aprendizaje, donde la interacción se produce no sólo entre alumno y materiales, o alumno y profesor, sino también entre los propios alumnos. Más aún, esta digitalización ha facilitado diseñar caminos de aprendizaje cooperativo entre los estudiantes, tanto información, materiales docentes, investigaciones o trabajos, e incluso ha abierto canales de evaluación que ha orientado en el avance del conocimiento de la materia, a través por ejemplo de plataformas de autoevaluación, simuladores, juegos educativos, foros temáticos, blogs especializados o redes sociales con espacios diseñados al aprendizaje.

b) Poner el foco sobre la creación de recursos y materiales didácticos que se convierte en el medio para desarrollar competencias como eje orientador de la práctica formativa de aprendizaje (Delors, 1996; González y Wagenaar, 2003), caracterizados por una visión radial (aplicables a distintos niveles formativos), multidisciplinar (distintas áreas, enfoques, metodologías y ámbitos de análisis), orientada a la síntesis (teoría y resolución de problemas prácticos y aplicaciones al entorno profesional) y actualizada (en continua revisión y adaptación). Apostar por la continua estimulación creativa en el desarrollo y presentación de los materiales docentes, pondera modelar los usos digitales (de la web 2.0 y 3.0) para crear procesos y entornos de aprendizaje con un carácter más social, dinámico, interactivo, creativo y personal (Shang, Li, Wu y Hou, 2011).

Estos pilares delimitan el optar por trabajar simultáneamente en el reducto (interno) del campus virtual y en plataformas sociales (sites, blogs, wikis, foros, etc.), que abren el aula al uso de recursos digitales que motiva, facilita y redirige a la acción docente a potenciar la creatividad del tándem profesor/alumno, en un espacio docente compartido, colaborativo, de responsabilidad mutua. En dicho espacio la coordinación es imprescindible y el profesor debe evaluar críticamente las ventajas del uso de las herramientas y recursos digitales, con formatos diversos y que contribuyan, lejos de ser resultado de un mero esnobismo o exhibicionismo didáctico, a favorecer el necesario tránsito desde el entorno docente al profesional, máxime cuando los estudiantes, tras un cuatrimestre de formación en el aula, realizan un prácticum en centros educativos (públicos, privados o concertados), como complemento tutorizado del aprendizaje de su oficio, en un entorno real. 
Pues bien, adentrarnos en la disciplina del self-study, ha supuesto reconocer la ventaja de ser un importante medio para mejorar la autocomprensión y propiciar la mejora de la relación entre la enseñanza y el aprendizaje sobre la enseñanza (Berry, 2016), desde la comprensión de nosotros mismos "self", tanto en el contexto de la actividad docente en el interior del aula, en colaboración con otros docentes y estudiantes, como en la interrelación con los estudiantes fuera del aula, construyendo conocimiento a partir de observar nuestro propio quehacer pedagógico, reflexionando, dialogando, valorando y verbalizando dicha observación. Es por tanto, una reflexión sobre necesidades, problemas, procesos de cambio, sentimientos de eficacia, creencias, etc., como factores que contribuyen al desarrollo profesional del propio docente, bien sea a través de saberes o prácticas nuevas o mejoradas (Avalos 2011).

Esto entronca con el planteamiento más extenso de la actividad de docentes (y estudiantes), que se convierten en investigadores de las experiencias propias y de sus compañeros, fortaleciendo así los vínculos entre docencia e investigación, y entre los Centros educativos y la Universidad (Ryan, 2014).

\subsection{Implantación y reflexión sobre el diseño de la estrategia ABPD: Self- study}

Moraes y Torre (2002) visualizan una educación no aferrada a la trasmisión de conocimientos fragmentados y disciplinarios, alejados de la realidad, que busque la globalidad y la interrelación de los saberes desde la implicación emocional. Así, esta multidisciplinariedad se puede ensamblar con otros tres elementos:

a) El desarrollo de herramientas validadas para el desarrollo y evaluación por competencias de los estudiantes: Para Brockbank y McGill (2002) los métodos de enseñanza aprendizaje tienen que potenciar que los alumnos aprendan a través de actividades que les permitan estructurar sus razonamientos, perspectivas, potencialidades y capacidad creativa, aportándoles una mayor capacidad de gestión del aprendizaje, siendo protagonistas de su propio proceso de aprendizaje. La asignatura pendiente es el diseño y validación de un modelo de medición del desarrollo competencial, indicador adicional de la calidad de los estudios universitarios en cuanto al potencial y capacidad de inserción laboral de los titulados (De Miguel, 2006 ; Marzo, Pedraga y Rivera, 2006).

b) La utilización de las analíticas del aprendizaje (learning analytic), que a partir del desarrollo de algoritmos asociados al uso de los rastros digitales dejados por los estudiantes, analizan de forma dinámica y en tiempo real el ritmo, frecuencias, patrones, y modalidades de aprendizaje, y definen perfiles de 
usos, patrones de navegación, pausas, hábitos de lectura y escritura, etc. (Ferguson, 2012; Siemens, 2013).

c) Ahondar en la experiencia de reflexión durante la formación inicial para ser docente, que para Perrenoud (2004) es necesaria y aumenta la comprensión de la actividad profesional, la complejidad de la enseñanza, mejora la habilidad para la innovación, estimula el aprendizaje a partir de la propia experiencia, y aporta significado, contextualizado a los componentes éticos, que lo configuran, y que conduce, necesariamente, a una serie de preguntas y líneas de investigación sobre nuestra propia acción docente.

Reflexionando sobre estos elementos, y teniendo en cuenta el aprendizaje de la reflexión sobre los antecedentes exhibidos, el ABPD, es una estrategia de aprendizaje que rediseña la simulación del ejercicio del futuro docente, donde los estudiantes experimentan con las diversas tareas propias de un docente, en las que desarrollarán habilidades que necesitarán para su carrera profesional, es decir, aprender su profesión haciendo, es decir, aprender a aprender y a enseñar, a la vez que genera una reflexión valorativa en su observador (el profesor y tutor).

La implantación de esta estrategia ABPD, del que el estudiante es conocedor metodológicamente desde el inicio el curso, ha requerido un proceso de ajuste durante más de una década, tanto a medida que nuevos profesores se incorporan a la misma, aportaban conocimientos, saberes propios y reflexiones complementarias, como por las iniciativas aportadas por los propios estudiantes. Por tanto, el ABPD se caracteriza por incidir en:

a) Alta motivación, creencia y compromiso con la estrategia para aplicarla sin fisuras: eso ha supuesto una cohesión en la forma de abordar la materia de didáctica por parte del equipo de docentes, dado que según el plan de estudios son dos asignaturas distintas (didáctica de ECO y didáctica de ADE).

b) Fuerte coordinación en los contenidos teóricos y en las actividades y recursos que se trabajan, y que requiere de un calendario temporal de trabajo, en una secuencia lógica y bien ensayada, que exigen que los profesores cumplan para favorecen la secuenciación definida.

c) Consistencia en la evaluación de los recursos y actividades, para lo cual se establecen unos criterios con elementos comunes, que responden a rúbricas que se readaptan a los mismos, y con flecos de flexibilidad.

d) Fomentar la incorporación al aula de los conocimientos de otras disciplinas o profesiones que aportan los propios estudiantes, por cuanto muchos de ellos llegan al MFP con una experiencia laboral muy enriquecedora, que se comparte con el grupo, bajo el criterio de aprender de nuestro bagaje e integrar conocimientos. 
e) Flexibilidad para adaptarnos a las necesidades e identidad concreta del grupo de estudiantes cada curso, creando un aula que respira y que se sale del corsé de un plan de estudios, en la que el docente está a su vez en continuo aprendizaje y experimentación, abierto a las iniciativas docentes.

Obviamente, formar a los futuros docentes, es una actividad que requiere asumir cierta responsabilidad, creer en el proyecto, estar altamente motivado, mostrarse tal cual, es decir, bajarse de la tarima y trabajar codo a codo dibujando el perfil de los futuros docentes, sin miedos, sin ambigüedades, aportando valores y empoderando la profesión de formador de formadores. También se reciben grandes satisfacciones, comprobando cómo crecen personal y profesionalmente nuestros estudiantes. Y desde esta óptica, surgieron una serie de preguntas que fueron enfocando los pasos para diseñar e implementar la estrategia que rige este caso:

- ¿Cuáles son las herramientas docentes más adecuadas dentro del aula? ¿Cuáles domino? ¿Cuáles puedo aprender?

- ¿Cómo puedo mejorar la interacción con las estrategias que utilizo en mi actividad? ¿Cuáles son las herramientas digitales mejores para interactuar con los estudiantes?

- ¿Cómo la reflexión crítica me permite ir mejorando en la estrategia docente? ¿Cómo incentivar a que los estudiantes reflexionen? ¿Cómo y cuándo yo hago mi propia reflexión deliberativa?

- ¿Cómo podemos automotivarnos? ¿Cómo podemos motivar a los futuros docentes? ¿Los alumnos pueden motivar al docente?

- ¿Debo compartir con los estudiantes la reflexión propia? ¿Debemos compartir las reflexiones significativas de los estudiantes?

Quizás para entender el proceso seguido sea necesario describir la estrategia ABPD implementada:

1. Contenido: Esta estrategia se aplica en el bloque temático de Didáctica de ECO y $A D E$, e interpretando lo especificado en la normativa educativa sobre el currículum de bachillerato y secundaria, elaboran un portafolio completo compuesto por: i) un proyecto didáctico realista y aplicable a un centro educativo, que se articula a partir de una programación docente (en grupo de 2 o 3 alumnos) y una unidad didáctica (individual). ii) una selección de los materiales o recursos elaborados secuencialmente durante el curso, que consideran más adecuados para su impartición en el aula y la evaluación de dicha unidad, con referencia a las competencias que sus futuros estudiantes podrían adquirir, y trabajando la transversalidad en los recursos y materiales; iii) una batería multiformato de pruebas de evaluación 
adaptada a la especifidad de sus posibles alumnos y las materias a impartir; iv) los ensayos de valoración y reflexión sobre los aspectos más destacables (positivos y negativos) del grupo de trabajo complementario del que actúan como evaluadores.

2. Evaluación según diversas rúbricas: El proyecto en su conjunto se evalúa en las diversas partes que lo componen: 25\% la Programación Didáctica, $25 \%$ la Unidad didáctica y $50 \%$ los recursos didácticos multiformato (póster, mapa conceptual, ficha de evaluación simulador o juego, video, micro investigación, trabajo desde el anuncio, técnicas de fijación, actividad fuera del aula, participación en el blog, etc.),

3. Aplicabilidad: La propuesta debe estar adaptada a contextos reales, particularizado a un centro educativo concreto, con la singularidad del área demográfica que se elija, y la composición del aula, tanto en identidades sociales como en tipología de estudiantes.

Por tanto, la estrategia se considera una adaptación del aprendizaje basado en problemas a la especifidad del entorno en el que se aplica (Boss y Krauss, 2007; Bender, 2012; Patton, 2012). Con temores similares a los expuestos por Kitchen (2005, 2016), a que sus estudiantes reproduzcan las técnicas pedagógicas tradicionales al carecer del repertorio de habilidades necesarias para aplicar enfoques consistentes, se integran conscientemente en el desarrollo de la ABPD actividades que modelen enfoques alternativos, de aprendizaje cooperativo, que comprometan a los estudiantes, en responsabilidad y control. Este proceso ha requerido un esfuerzo continuado de coordinación con los profesores de cuatro departamentos y disciplinas distintas que intervienen en el bloque temático de didáctica (ECO y ADE).

Todas las actividades y recursos que los estudiantes realizan durante el curso son evaluados (valoración inicial por el propio estudiante en el aula, por reflejo con otros, y por el profesor), para lo cual se ha adaptado una rúbrica a los contenidos del curso (cuadro 1), definida a partir de Villalustre y del Moral (2014). Llegar a esta rúbrica ha sido a su vez un proceso largo de ajustes y en algunas ocasiones de prueba y error al tratar de cuantificar aspectos difíciles de evaluar, que ha requerido una valoración más cualitativa que cuantitativa. Los estudiantes participan en la concreción de la misma, así como en la asignación de ponderaciones. Obviamente todos estos recursos deben cumplir con los criterios de creatividad-digitalizaciónoportunidad, ser válidos para el aprendizaje y versátiles.

El bloque de recursos a trabajar, perfectamente preparado, ensayado y autoevaluado, proviene de una amplia experiencia del docente con estudiantes de perfiles formativos muy diversos, que responden de forma similar a los mismos estímulos 
motivacionales, siendo la sesión inicial un choque metodológicoª a partir de tres elementos diseñados ex profeso para cumplir el objetivo, y que constituyen la primera etapa de la reflexión a la que se somete a los estudiantes en interacción con el profesor.

1. Encuesta sobre los atributos docentes, que consideran más relevantes en un profesor.

2. Planteamiento metodológico del trabajo docente, motivado por preguntas básicas que entroncan con el proceso de aprendizaje seguido desde la infancia: reconocer lo que se sabe y avanzar en el conocimiento, desde la abstracción, progresando en grado de dificultad.

3. Presentación de recursos digitales y creativos, que a partir de la descripción sumatoria del grupo se trabajan las distintas dimensiones derivadas de las respuestas individuales, y en diversos soportes.

Así, Robin (2008) destaca la carga expresiva de combinar imágenes gráficas, ilustraciones, dibujos, mapas, animaciones, música, por cuanto que el despliegue creativo que conlleva le convierte en una interesante estrategia en los diversos contextos educativos, ya que permite transmitir ideas, información o mensajes de forma directa y sintética, y supone la puesta en práctica de competencias diversas.

El resto del curso se usan diversos recursos, medios materiales y espacios, donde la pizarra se transforma en un lienzo, los muros del aula en tablones para acoger lluvias de ideas, pósteres, recortes de prensa, etc. y el suelo es un área de trabajo grupal: cualquier espacio físico y medio material es válido si es útil para conseguir el objetivo de comunicar eficazmente un mensaje claro y bien elaborado.

El trabajo en el aula es previo al trabajo fuera de ella, bien sea individual (excepcionalmente), colectivo y grupal (mayoritariamente), donde todos (profesor y estudiantes) son responsables de la calidad del mismo y del aprendizaje individual y colectivo (Slavin, 1999), según los objetivos prefijados. El trabajo en el aula reconoce aspectos teóricos básicos, identifica objetivos y prácticas validadas o con posibilidades de mejora, y define un mensaje unívoco: se trabaja y reflexiona el acierto para reforzarlo y el error para identificar vías de superación.

\footnotetext{
${ }^{9}$ Los asistentes conocen el área de formación y especialización del docente ("¿qué hace un profesor de Análisis Económico dando didáctica?")
} 
Cuadro 1. Esquema de la rúbrica de evaluación de los recursos didácticos

\begin{tabular}{|c|c|c|c|}
\hline & \multicolumn{3}{|c|}{ Nivel de competencia } \\
\hline & Bajo & Medio & Alto \\
\hline $\begin{array}{l}\text { 1. Usos } \\
\text { digitales }\end{array}$ & $\begin{array}{l}\text { Escaso uso multiformatos. } \\
\text { Gestión baja de } \\
\text { componente formativo }\end{array}$ & $\begin{array}{l}\text { Adecuada utilización de } \\
\text { formatos digitales. } \\
\text { Uso de diversoso soportes }\end{array}$ & $\begin{array}{l}\text { Elevado uso de } \\
\text { herramientas digitales y } \\
\text { diversas. } \\
\text { Buena selección y } \\
\text { adecuación a contenidos }\end{array}$ \\
\hline $\begin{array}{l}\text { 2. Habilidades } \\
\text { expositivas }\end{array}$ & $\begin{array}{l}\text { Poco adecuada o fluida. } \\
\text { Escasa secuencia } \\
\text { expositiva. } \\
\text { Incoherencias en la } \\
\text { presentación }\end{array}$ & $\begin{array}{l}\text { Adecuada temática. } \\
\text { Adecuada secuencia } \\
\text { expositiva. } \\
\text { Adecuada presentación }\end{array}$ & $\begin{array}{l}\text { Temática claramente } \\
\text { definida. } \\
\text { Buena secuencia } \\
\text { expositiva. } \\
\text { Coherencia entre contenido } \\
\text { y presentación }\end{array}$ \\
\hline $\begin{array}{l}\text { 3. Aporte } \\
\text { creativo }\end{array}$ & $\begin{array}{l}\text { Escasa creatividad en los } \\
\text { recursos digitales }\end{array}$ & $\begin{array}{l}\text { Uso de diferentes recursos } \\
\text { digitales. } \\
\text { Capacidad expresiva } \\
\text { limitada }\end{array}$ & $\begin{array}{l}\text { Potencia la capacidad } \\
\text { expositiva. } \\
\text { Originalidad e innovación } \\
\text { de los recursos. }\end{array}$ \\
\hline $\begin{array}{l}\text { 4. Aspectos } \\
\text { didácticos }\end{array}$ & $\begin{array}{l}\text { Esxcaso potencial } \\
\text { didáctico. } \\
\text { Confusión en la transmisión } \\
\text { de conocimientos. }\end{array}$ & $\begin{array}{l}\text { Exposición adecuada y } \\
\text { motivadora. } \\
\text { La estrategia didáctica } \\
\text { puede ser mejorable. }\end{array}$ & $\begin{array}{l}\text { Potencioa la reflexión y } \\
\text { adquisición de valores. } \\
\text { Uso de estrategias } \\
\text { adecuadas. }\end{array}$ \\
\hline $\begin{array}{l}\text { 5. Interacción } \\
\text { del grupo }\end{array}$ & $\begin{array}{l}\text { Escasa interacción. } \\
\text { Baja aportación en las } \\
\text { entradas y documentos }\end{array}$ & $\begin{array}{l}\text { Adecuada interacción con } \\
\text { el grupo. } \\
\text { Entradas y documentos } \\
\text { bastantes significativos }\end{array}$ & $\begin{array}{l}\text { Elevada participación e } \\
\text { interacción. } \\
\text { Entradas con contenido } \\
\text { significativo y concluyente }\end{array}$ \\
\hline \multicolumn{4}{|c|}{ Rúbrica interacción del Grupo } \\
\hline $\begin{array}{l}\text { Aspectos } \\
\text { formales }\end{array}$ & $\begin{array}{l}\text { Plazos frecuencia } \\
\text { Cumplimiento de plazos. } \\
\text { Intervenciones regulares }\end{array}$ & $\begin{array}{l}\text { Autoría } \\
\text { Autoría: copia, plagio. } \\
\text { Aspectos ortográficos, } \\
\text { redacción. }\end{array}$ & $\begin{array}{l}\text { Aportaciones extras } \\
\text { Documentación adicional: } \\
\text { recursos, enlaces, artículos, } \\
\text { ejemplos. } \\
\text { Referencias bibliográficas } \\
\text { y citas. }\end{array}$ \\
\hline $\begin{array}{l}\text { Aspectos } \\
\text { concretos }\end{array}$ & $\begin{array}{l}\quad \text { Cumplimiento } \\
\text { Responden a las } \\
\text { intervenciones. } \\
\text { Plantean nuevas ideas o } \\
\text { líneas }\end{array}$ & $\begin{array}{l}\quad \text { Calidad } \\
\text { Calidad de los materiales. } \\
\text { Relación con el tema de } \\
\text { trabajo. }\end{array}$ & $\begin{array}{l}\text { Evaluación y feed-back } \\
\text { Aporta feed-back a sus } \\
\text { compañeros. } \\
\text { Evalúan significativamente. }\end{array}$ \\
\hline
\end{tabular}

Esta propuesta didáctica permite a los futuros docentes, ejercitar o desarrollar distintas competencias ligadas a la implementación curricular en los entornos profesionales de los centros de educación secundaria y de formación profesional. Los estudiantes la evalúan en dos momentos claves del curso: al comienzo y al 
final, para cuantificar cambios en la graduación, la importancia y sus reflexiones de entrada y salida (detalle en el cuadro 2). En este camino el profesor acompaña a los estudiantes con su autorreflexión y valoración.

Cuadro 2. Referencia a competencias, desarrollo y evaluación

\begin{tabular}{|c|c|}
\hline Competencias profesionales del docente & Reflexión valorativa cualitativa \\
\hline Capacidad para aplicar técnicas digitales multiformato & $\begin{array}{l}\text { Deconocimiento, técnicas } \\
\text { Dificultad ritmos y estilos docentes. }\end{array}$ \\
\hline $\begin{array}{l}\text { Capacidad para diseñar, planificar y organizar una estrategia } \\
\text { docente con recursos multiformato. }\end{array}$ & $\begin{array}{l}\text { Deficiencias pedagógicas } \\
\text { Desconocimiento planificación } \\
\text { Inseguridad aplicación } \\
\text { Desajustes ritmos }\end{array}$ \\
\hline $\begin{array}{l}\text { Capacidad para desarrollar recursos multiformato con usos } \\
\text { educativos y formativos }\end{array}$ & $\begin{array}{l}\text { Inseguridad usos } \\
\text { Bajos niveles de creatividad } \\
\text { Inseguridad } \\
\text { Deescreencia }\end{array}$ \\
\hline $\begin{array}{l}\text { Habilidad para evaluar recursos multiformato disponibles, } \\
\text { para su uso en entornos educativos y formativos. }\end{array}$ & $\begin{array}{l}\text { Deficiencias pedagógicas } \\
\text { Baja creatividad } \\
\text { Inseguridad } \\
\text { Visión tradicional }\end{array}$ \\
\hline Habilidad para aplicar las TIC en una estrategia docente & $\begin{array}{l}\text { Inseguridad } \\
\text { Dificultad ritmos y estilos docentes } \\
\text { Visión tradicional }\end{array}$ \\
\hline Competencias genéricas y transversales & Reflexión valorativa cualitativa \\
\hline Capacidad de busqueda de información relevante & $\begin{array}{l}\text { Buen desempeño } \\
\text { Seguridad } \\
\text { Requiere tiempo y filtrar información }\end{array}$ \\
\hline Capacidad para generar y gestionar información multiformato & $\begin{array}{l}\text { Interés y creatividad } \\
\text { Requiere tiempo y filtrar información }\end{array}$ \\
\hline Capacidad para plantear ideas innovadoras y creativas & $\begin{array}{l}\text { Inseguridad } \\
\text { Dudas pedagógicas } \\
\text { Dificultades defensa y exposición }\end{array}$ \\
\hline $\begin{array}{l}\text { Capacidad de evaluación critica de materiales disponibles } \\
\text { (pensamiento crítico) }\end{array}$ & $\begin{array}{l}\text { Inseguridad } \\
\text { Duddas pedagógicas } \\
\text { Baja creatividad } \\
\text { Visión radial }\end{array}$ \\
\hline Capacidad para tomar decisiones (en un entormno formativo) & $\begin{array}{l}\text { Inseguridad } \\
\text { Responsabilidad } \\
\text { Información }\end{array}$ \\
\hline Capacidad para trabajar en equipo & $\begin{array}{l}\text { Cómodos } \\
\text { Necesario } \\
\text { Generador conocimiento }\end{array}$ \\
\hline Habilidad para incrementar la sensibilidad estética & $\begin{array}{l}\text { Interesante } \\
\text { Inseguridad }\end{array}$ \\
\hline
\end{tabular}




\subsection{Reflexión final y observación en la implementación de la estrategia ABPD: post self-study}

Adentrarse y aplicar la disciplina del self-study ha permitido, siguiendo a Santibanez y otros (2017) comprender y construir relaciones, con nuestros estudiantes, colegas y con nosotros mismos; entrar en una revisión sistemática y rigurosa sobre la formación que damos a futuros docentes que cambia nuestro punto de vista sobre el hacer docente, conectando la investigación y la docencia; ahondar en las discusiones sobre los métodos de investigación que vinculan la construcción de conocimiento con los procesos formativos, adentrándonos en otras líneas y metodologías; ampliar la sensibilidad para atender los aprendizajes que derivan de la propia experiencia acentuando la honesta reflexividad pedagógica, aceptar que se quiere ser mejor docente, reconociendo los fallos o debilidades y abordando acciones de mejora, que consoliden las fortalezas.

Este camino no se ha realizado solo, se ha compartido, visibilizando reflexiones, recogiendo la colaboración y el feedback de compañeros (Pithouse-Morgan y otros, 2015 se refieren a la reflexión colaborativa o co-reflexiva, conociendo el proceso de reflexión que están viviendo los otros), y estudiantes (que han ido construyendo una matriz de preguntas que responder requería adentrarse en nuevas reflexiones pedagógicas constructivas), apoyándonos principalmente en las aportaciones del "acompañante crítico" que, ocupando una posición fundamental en la conversación reflexiva, nos hace cuestionarnos y profundizar en la construcción del aprendizaje basado en nuestra experiencia y construir un saber pedagógico (Pinnegar y Hamilton, 2009). Dicho acompañante crítico (Olan y Edge, 2019) adopta el rol de provocarnos la discusión reflexiva y valorativa, pero también de oyente, consejero o acompañante, que nos respeta y no nos juzga, en espacios de apoyo, comprensión y aprendizaje mutuo. Este acompañamiento, consciente o no, garantiza el crecimiento como docente, es decir, co-aprendiz de una profesión (Iglesia, 2012). Aquí es cuando aludimos al concepto del carácter relacional del self-study que construye una comunidad reflexiva, generando con otros y en torno a otros una relación empática, en especial con nuestros estudiantes y futuros docentes y el acompañante crítico.

Obviamente, las reflexiones del docente interactúan continuamente con las de los estudiantes, motivando el ejercicio de reflexión, abriendo canales de confianza e interrelación, exhibiendo las propias dudas, intereses, dificultades experimentadas, necesidades de profundizar en aspectos pedagógicos o metodológicos, así como con las aportaciones extraídas del catálogo de documentos coleccionados y temas explorados en las entrevistas semi estructuradas. 
La adaptación de los estudiantes al método de trabajo activo, colaborativo y reflexivo requiere un esfuerzo continuado, suyo y del docente, individual y colectivo. La valoración que realizan los estudiantes sobre el método de trabajo es positiva (véase cuadro 3, referido a los datos del curso 2017-18), por cuanto que les permite acercarse a la profesión docente desde diversos frentes: Tener una visión más integrada de la profesión docente $(4,1)$, Analizar situaciones de práctica profesional $(4,1)$, Desarrollar competencias necesarias en la práctica profesional $(4,3)$. Este hecho que se refleja en las elevadas calificaciones que obtienen, si bien a costa de un esfuerzo más continuado, lejos del trabajo puntual más tradicional en otras etapas formativas, exigiéndoles mayor compromiso, que les lleva a valorar la acción docente en diversas dimensiones. Así, la valoración general del ABPD $(4,0)$, el proyecto ha mejora el proceso de enseñanza-aprendizaje $(4,1)$, el sistema de valuación ha sido apropiado $(4,3)$ y las orientaciones del profesor han sido adecuadas $(4,2)$. Se pone de manifiesto la necesidad de un mayor esfuerzo y continuado, la orientación profesional, profundizar en aspectos metodológicos, mejora la confianza, motivación y autoestima, visión más radial, reduce inseguridades, mayor fluidez y conocimiento del entorno.

Una dificultad de la aplicación del ABPD es la secuenciación en las sesiones de trabajo (en el primer trimestre del curso se concentran los bloques de didáctica y el resto de materias curriculares), que exige avanzar en las entregas de los materiales o recursos elaborados para su exposición y evaluación por el profesor y colectivamente, e intensifica el trabajo del estudiante y docente. Otro aspecto de mejora es afinar en la detección de las aportaciones desiguales en la calidad del trabajo en las entregas o dinámicas grupales, para lo cual se debe implementar un instrumento de co-evaluación entre los integrantes del grupo que pueda generar calificaciones diferentes intra-grupo, si bien los estudiantes tienen opiniones contradictorias al respecto.

Como reflexión final, el docente que aplica esta metodología necesita estar en continua renovación y actualización, adaptando los soportes, las dinámicas y los recursos digitales en función de la naturaleza del grupo de estudiantes, sus conocimientos previos y la experiencia que revierten al aula, e incorporando aquellos elementos de comunicación social y de la web 3.0 que son habituales en el entorno de sus estudiantes, para ser moldeados como recurso docente aplicable al aula. 
Queda claro que, al igual que Zeichner y Noffke (2001), McWilliam (2004) y Snow-Gerono (2005), la motivación del docente para participar en este tipo de investigación incluye un interés por comprender mejor a los alumnos, mejorar la práctica docente, y generar conocimiento, sobre la enseñanza y la mejora de los contextos en los que se integra la práctica educativa.

Cuadro 3. Elementos de Valoración de la ABPD (entre 1 y sobre 5)

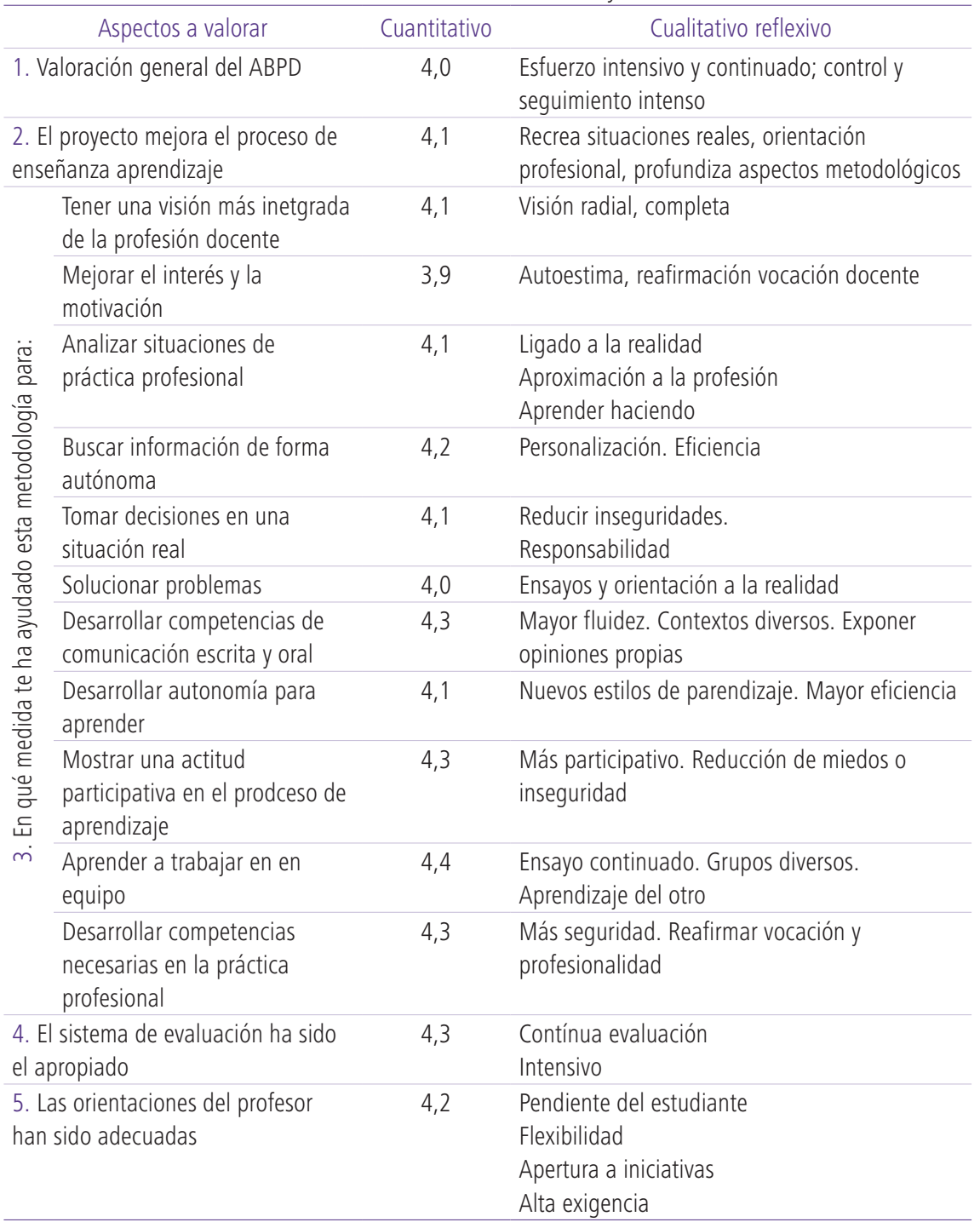


Una aclaración final necesaria es que la concentración de materias curriculares en el primer trimestre viene determinada por el plan de estudios verificado del MFP, y permite que en el segundo los estudiantes completen su formación en las prácticas externas que realizan en institutos y centros educativos. En este periodo de prácticas pondrán en práctica lo trabajado en el aula, generando así su propia reflexión formativa, mejorando su competencia profesional y gestión de emociones, tal como exponen García-Lázaro, Colás-Bravo y Conde-Jiménez (2020), en la línea de Jackson (2017), Thomas (2017) y Ripamonti, Galuppo, Bruno, Ivaldi y Scaratti (2018).

\section{Discusión final y conclusiones}

Recogiendo la definición de self-study de Hamilton y Pinnegar (1998) como el estudio de uno mismo, nuestras acciones e ideas, el ejercicio realizado es un compendio de trazos autobiográficos, del contexto social y cultural, que dibujan nuestra propia vida académica e implica una mirada atenta a los textos leídos, las experiencias y las personas conocidas. El futuro puede modificar el presente reorganizándolo tan rápido como sucede, y a la vez debemos utilizar el presente para entender la práctica docente que ejerceremos, entrando en un diálogo en continuo equilibrio con nuestro pasado y futuro como docentes (Stern, 2004), tal como se ha mostrado, por cuanto el presente no se entiende sin el pasado y el futuro, y condiciona a su vez al futuro ejercicio docente, en una continua revisión y reflexión de la acción docente. Se refiere dicho autor a que las exploraciones del presente y del futuro revierten en la conciencia del conocimiento implícito, caracterizado por ausencia de simbolismos, no verbal, inconsciente, construido en el presente en respuesta a nuestras experiencias, rico y profundo, que incluye los afectos, las expectativas, los cambios en la motivación y la acción, así como las orientaciones al mundo y los estilos de pensamiento, y que seguramente es la fuente de las interrelaciones con los demás, estudiantes y colegas.

Con ese guión, hemos transitado por el diseño de una estrategia metodológica, su implementación en el aula y la investigación sobre la misma, con elementos innovadores y/o creativos, tratando de aunar diversos atributos como la digitalidad, el aprendizaje activo, y el desarrollo de diversas competencias tanto genéricas y transversales como sociales y emocionales, que favorezcan una maduración intelectual y profesional en el propio docente y en sus estudiantes y futuros docentes, en un continuo acercamiento al ejercicio profesional al aprender (o reaprender) la profesión. La experiencia diseña una estrategia de aprendizaje radial, multidis- 
ciplinar, orientada a la síntesis, actualizada, que abre el aula al uso de recursos que motivan, facilitan, redirigen y potencian la creación de un espacio docente compartido, que sintetiza la metodología Aprendizaje Basado en un Proyecto Docente (ABPD).

Se considera que esta estrategia genera resultados colaterales, cuyo valor añadido está en que I) los estudiantes desarrollan diversas competencias básicas en el entorno profesional al que se insertarán; adoptan la responsabilidad en la elaboración de materiales docentes de calidad compartidos, evalúan a sus compañeros con espíritu crítico con afán de mejora, etc. y II) el docente ahonda en la interrelación con su actividad, la de sus colegas y estudiantes, continuando en el bucle de la reflexión construyendo conocimiento pedagógico y metodológico.

Este camino no se ha realizado en soledad, se ha ido acompasando a la formación en nuevas disciplinas y la incorporación de metodologías y TICs, fruto tanto de la propia convicción de sus ventajas en el entorno educativo actual, como de la rápida adaptación del alumnado a los nuevos modos de aprendizaje, que en muchos casos ha ido un paso por delante, motivando e incentivando al docente, en su apuesta decidida de renovación reflexiva del oficio.

Numerosas han sido las preguntas que han guiado el trabajo docente y la reflexión consciente sobre la práctica, que suelen quedar abiertas y en continua reverberación: ¿Qué me interesa explorar sobre el ABPD? ¿Qué elementos identifico como problemas en mi práctica? ¿Detecto contradicciones entre mis opiniones, valores y acciones? ¿Qué quiero aprender sobre mis intereses y preocupaciones y cómo explorarlos? ¿EI ABPD es un contexto adecuado para la formación de docentes? ¿Qué elementos/métodos puedo mejorar o incorporar? ¿Cuáles son las evidencias y experiencias a rescatar? ¿Qué trabajo en investigación puede guiar mi introspección? ¿Qué valores personifico en mi práctica e investigación? ¿Qué espero aportar a mi ejercicio profesional? Haciendo un símil, si Long y Siemens (2011) consideran que el análisis del aprendizaje (learning analytic) está permitiendo ver, entre la niebla de la incertidumbre, para instrumentar mejoras en la calidad y el valor de la experiencia personalizada de aprendizaje, tanto para el docente como los estudiantes, la práctica del self-study está permitiendo navegar por el interior de la conciencia y el corpus del docente en acción, conociendo sus reflexiones, miedos, pasiones e inseguridades. 
El camino reflexivo seguido ha sido largo, y ha supuesto para el docente mostrarse sin coberturas, pero también reafirmarse en su visión de ser co-aprendiz de su profesión (Iglesia, 2018), con el doble objetivo de transmitir conocimiento derivado de la práctica en el ejercicio profesional y ser un acompañante en el proceso formativo de los futuros docentes. No obstante, Murray y Male (2005) inciden en que los formadores de docentes llegan a conforman un grupo académico de expertos que se convierten en novatos ante la necesidad de adquirir las nuevas y amplias habilidades pedagógicas que exige la formación de profesores. Sí es cierto que poder observar a los futuros docentes en acción, tanto en la propia Universidad como en los centros en los que se insertan, y aportarles una valoración crítica constructiva, supone un desafío continuo por cuanto que pueda reorientar los supuestos fundamentales de la práctica profesional personal, y la comparativa con uno mismo es inevitable, salvando las distancias de experiencias, entornos vividos, responsabilidades, objetivos o motivaciones. Esta observación y reflexión contribuye a regenerar los conocimientos propios como docente de futuros profesores, reorientar las propuestas pedagógicas y emprender acciones de mejora, cuestionando nuestro propio quehacer en un crecimiento continuo.

Reconocer la presión que se siente al querer ser ese acompañante, buen docente, llegar con un mensaje claro y sin ambigüedades y convertirse en un referente, puede provocar ciertas tensiones tanto emocionales como en el desempeño de los roles adoptados, si bien el ejercicio de self-study proporciona una poderosa vía de desarrollo profesional para los formadores de profesores/investigadores, desde la experiencia y el aprendizaje continuado.

Se han apuntado algunos elementos que responden parcialmente a las preguntas planteadas por Maloney y Konza (2011) sobre los procesos a seguir para desarrollar una comunidad de aprendizaje profesional eficaz, y los factores que impactan en el desarrollo de un aprendizaje profesional efectivo, en colaboración con los futuros profesores. Quedan abiertas, por tanto, abordar algunas acciones de mejora en la formación de los docentes, en el propio aula o en los centros en los que realizan las prácticas (Rusell, 2017; MacKinnon, 2017), así como nuevas líneas de investigación.

\section{Referencias bibliográficas}

Avalos, B., (2003). Teacher professional development in Teaching and Teacher Education over ten years, Teaching and Teacher Education, 27, 10-20. 
Bender, W. (2012). Project-Based Learning: Differentiating Instruction for the $21^{\text {st }}$ Century. California: Corwin.

Berry, A. (2016). Tensiones en la reconceptualización del conocimiento del formador de formadores: explorando la tensión entre valorar y reconstruir la experiencia. En: Russell, Hirmas y Fuentealba (Eds.), Formadores de formadores, descubriendo la propia voz a través del self-study (pp. 77-95). Santiago de Chile: OEl.

Boss, S. y Krauss, J. (2007).Reinventing Project-Based Learning: your field-guide to real-word projects in the Digital Age. Washington-EEUU: ISTE.

Brockbank A. y Mc Gill I. (2002). Aprendizaje reflexivo en la Educación Superior. Madrid, España: Ediciones Morata.

Cabrera-Murcia, E.P., Silva, C. y Gorichón, S. (2020). Formas de enseñar que favorecen en mis estudiantes la reflexión en torno a cómo brindar en la primera infancia experiencias de enseñanza potenciadoras de aprendizaje: una mirada desde mi práctica docente. Revista Iberoamericana de Educación, 82(1), 75-97. https:// doi.org/10.35362/rie8213650.

Carrasco, A., Gracia, E., e Iglesia, M. C. (2004). Hacia una nueva estrategias didáctica. Tres experiencias docentes con TIC en la enseñanza de Teoría Económica", en Libro de actas de la XIV Jornadas Luso-Españolas de Gestión Científica. Ed. Universidad de Ponta Delgada.

Conde-Jimenez, J. y Martín-Gutierrez, A. (2016). Potencialidades y necesidades de mejora en la formación de profesores noveles universitarios. Revista Electrónica de Investigación Educativa 18(1), 140-152.

Delors, J. (1996). La educación encierra un tesoro. Madrid: Unesco-Santillana.

Dondi, C. (2001). El desarrollo del individuo, el trabajador, el ciudadano: los objetivos de la educación en la sociedad de la información: cómo pueden ayudar las TIC a la innovación". Scienter. https://bit.ly/360YM12

De Miguel, M. (2006). Métodos y metodologías de enseñanza en la educación superior. Metodologías de enseñanza y aprendizaje para el desarrollo de competencias. De Miguel (Coord.), Madrid. Alianza editorial.

Echevarría, B. y Martínez, P. (2018). Revolución 4.0, Competencias, Educación y Orientación. RIDU: Revista digital de investigación en docencia universitaria, 12(2). https:// doi.org/10.19083/ridu.2018.831

Ferguson, R. (2012). Learning analytics: drivers, developments and challenges. International Journal of Technology Enhanced Learning, 4(5/6), 304-317.

García-Lázaro, I., Colás-Bravo, P. y Conde-Jiménez, J. (2020). Análisis de las necesidades formativas del tutor universitario de prácticas externas desde la perspectiva del self-study. Revista Iberoamericana de Educación, 82(1), 121-140. https://doi. org/10.35362/rie8213652.

Gilbert, M. (2001). El profesor del siglo XXI: de transmisor de contenidos a guía del ciberespacio. Dis'ponible en https://bit.ly/2OqW4IH. 
González, J. y Wagenaar, R. (2003). Tuning Educational Structures in Europe. Informe Final Fase Uno. Bilbao: Universidad de Deusto.

Hamilton, M. L. \& Pinnegar, S. (1998). Introduction: Reconceptualizing teaching practice. In M. L. Hamilton, S. Pinnegar, T. Russell, J. Loughran, y V. LaBoskey (Eds.), Reconceptualizing the education of teachers: Self-study in teacher education, 1-4. London: Falmer.

Harrison, C. y Killion, J. (2007). Ten roles for teacher leaders. Educational Leadership, 65(1), 74-77. Disponible en https://bit.ly/396MTVL.

Iglesia, M. C. (2012). La identificación de factores en el desarrollo de competencias de los estudiantes universitarios. Un estudio exploratorio. Revista Complutense de educación, 23(1), 207-240. Disponible en https://bit.ly/36UndKr

Iglesia, M. C. (2018). Aprendizaje Basado en un Proyecto Docente: Aprendizaje, creatividad, innovación y nuevos roles en la formación de profesorado en la era digital. Revista Complutense de Educación 29(4), 1253-1278. Disponible en https://bit.ly/393StrL

Johns, C. (2010). Guided reflection: A narrative approach to advancing professional practice. Oxford: Wiley-Blackwell.

Jackson, D. (2017). Developing pre-professional identity in undergraduates through workintegrated learning. Higher Education, 74(5), 833-853. https://doi.org/10.1007/ s10734-016-0080-2.

Kitchen, J. (2005). Looking Backward, Moving Forward: Understanding my Narrative as a Teacher Educator, Studying Teacher Education, 1(1), 17-30. https://doi. org/10.1080/17425960500039835

Kitchen, J. (2016). Mirando al pasado, avanzando hacia el futuro: comprendiendo mi narrativa como formador de formadores. En: Russell, Hirmas, y Fuentealba (Eds.), Formadores de formadores, descubriendo la propia voz a través del self-study, 189-203. Santiago de Chile: OEI.

LaBoskey, V. K. (2004). The Methodology of Self-Study and Its Theoretical Underpinnings. En: Loughran, Hamilton, LaBoskey y Russell (Eds.), International Handbook of Self-Study of Teaching and Teacher Education Practices (pp. 817-869). Springer Netherlands. https://doi.org/10.1007/978-1-4020-6545-3 21

Long, Ph. y Siemens, G. (2011). "Penetrating the Fog: Analytics in Learning and Education". EDUCAUSE Review, 46(5), 30-40.

Loughran, J. y Russell, T. (2016). Comenzando a entender la enseñanza como una disciplina. En: Russell, Hirmas, y Fuentealba (Eds.), Formadores de formadores, descubriendo la propia voz a través del self-study, 65-76. Santiago de Chile: OEI.

MacKinnon, A. (2017). Practicum and Teacher Education: Wrapped Around Your Finger. Studying Teacher Education, 13(2), 231-238. https://doi.org/10.1080/1742596 4.2017.1342361.

McWilliam, E. (2004). W(h)ither practitioner research? The Australian Educational Researcher, $31(2), 113-126$.

Maloney, C. y Konza, D. (2011). A case study of teachers' professional learning: Becoming a community of professional learning or not? Issues in Educational Research, 21(1). 
Marzo, M., Pedraja, M. y Rivera, P. (2006). Habilidades y competencias demandadas por el mercado laboral. Icade: Revista de las Facultades de Derecho y Ciencias Económicas y Empresariales, 73, 347-363.

Moraes, M. C. y Torre, S. D. L. (2002). Sentipensar bajo la mirada autopoética o cómo reencantar creativamente la educación. Creatividad y sociedad, 2, 41-56.

Murray, J. y Male, T. (2005). Becoming a teacher educator: Evidence from the field. Teaching and teacher education, 21(2), 125-142.

Official Journal of the European Union (2008). Decisión No 1350/2008/EC OF European Parliament and of the Council, of 16, december.

Olan, E. L. y Edge, C. (2019) Collaborative Meaning-Making and Dialogic Interactions in Critical Friends as Co-Authors. Studying Teacher Education, 15(1), 31-43. https://doi.or $\mathrm{g} / 10.1080 / 17425964.2019 .1580011$

Patton, A. (2012). Work that matters. The Teacher's guide to project-based learning. Londres: PaulHamlynFoundation. Disponible en https://bit.ly/2v4W7TS

Pascu, C. (2008). An Empirical Analysis of the Creation, Use and Adoption of Social Computing Applications. IPTS Exploratory Research on the Socio-economic Impact of Social Computing. Institute for Prospective Technological Studies (IPTS), JRC, European Commission. EUR 23415. Disponible en https://bit.ly/2u8p5lH

Perrenoud, P. (2004). Desarrollar la práctica reflexiva en el oficio de enseñar: Profesionalización y razón pedagógica. México: Colofón.

Pinnegar, S. y Hamilton, M. L. (2009). Self-Study of Practice as a Genre of Qualitative Research: Theory, Methodology, and Practice. Netherlands: Springer. Series: Self-Study of Teaching and Teacher Education Practices, 8.

Pithouse-Morgan, K., Muthukrishna, N., Pillay, D., Van Laren, L., Chisanga, T., Meyiwa, T. y Stuart, J. (2015). Learning about Co-Flexivity in a Transdisciplinary Self-Study. Research Supervision Community. En: Pithouse-Morgan y Samaras (Eds.), Polyvocal Professional Learning through Self-Study Research, 145-171. Netherlands: Sense Publishers. https://doi.org/10.1007/978-94-6300220-2 9.

Ripamonti, S., Galuppo, L., Bruno, A., Ivaldi, S. \& Scaratti, G. (2018). Reconstructing the internship program as a critical reflexive practice: the role of tutorship. Teaching in Higher Education, 1-18. https://doi.org/10.1080/13562517.2017.1421627.

Robin, B. (2008). Digital storytelling: a powerful technology tool for the $21^{\text {st }}$ century classroon. Theory into practice, 47(3), 220-228.

Robinson, J. (1974). Ensayos sobre Análisis Económico. Madrid: Fondo de Cultura Económica de España.

Russell, T. Fuentealba, R. e Hirmas C. (2016) (Eds.). Formadores de formadores, descubriendo la propia voz a través del self-study, Santiago de Chile: OEl.

Russell, T. (2017). A teacher educator's lessons learned from reflective practice. European Journal of Teacher Education, 41(1), 4-14. https://doi.org/10.1080/02619768 .2017 .1395852 . 
Ryan, Ch. (2014). Becoming Teachers, Becoming Researchers: a Case Study. American Journal of Educational Research, 2(8), 585-591. Disponible en https://bit.ly/36XWRqO.

Samaras, A. P. (2002), Self-Study for Teacher Educators: crafting a pedagogy for educational change. New York: Peter Lang Publishing.

Santibanez, D., Silva-Peña I., Tello P., Flores M., Nocetti A., Gutiérrez M. y Orrego C. (2017). Selfstudy como proceso de formación de formadores, pp. 115-136, en Investigación para la formación de profesores. Ediciones Universidad Católica Silva Henríquez, Eds González-García; Silva-Peña; Sepúlveda-Parra; Del Valle.

Schön, D. (1998). El profesional reflexivo. Cómo piensan los profesionales cuando actúan. Barcelona: Paidós.

Schumpeter, J. A. (1954). History of Economic Analysis. (First published 1954 por Allen \& Unwin Publishers Ltd); Reeditado por Taylor \& Francis e-Library, 2006.

Shang, S., Li, E.Y., Wu, Y.L. y Hou, O. (2011). Understanding web 2.0 service models: a knowledge-creating perspective. Information \& Management, 48, 178-184.

Siemens G. (2013). Learning Analytics: The Emergence of a Discipline, American Behavioral Scientist, 57(10), 1380-1400, SAGE Publications. Disponible en https://bit. Iy/2GR971R.

Slavin, R. (1999). Aprendizaje cooperativo. Teoría, investigación y práctica. Argentina: Aique.

Snow-Gerono, J., (2005). Professional development in a culture of inquiry: PDS teachers identify the benefits of professional learning communities. Teaching and Teacher Education, 21(3), 241-256.

Stern, D. (2004). The present moment in psychotherapy and everyday life. New York: W.W. Norton \& Company.

Terigi, F. (2012). Los saberes docentes. Formación, elaboración en la experiencia e investigación. Buenos Aires: Fundación Santillana.

Thomas, L. (2017): Learning to Learn about the Practicum: A Self-Study of Learning to Support Student Learning in the Field. Studying Teacher Education, 13(2), 165-178. https:// doi.org/10.1080/17425964.2017.1342354.

Villalustre, L y del Moral M. E. (2014). Digital storytelling: una nueva estrategia para narrar historias y adquirir competencias por parte de los futuros maestros. Revista Complutense de Educación, 25(1), 115-132. http://revistas.ucm.es/index.php/ RCED/article/view/41237

Wake, J. Dysthe, O. y Mjelstad, S. (2007). New and Changing Teacher Roles in Higher Education in a Digital Age. Educational Technology and Society, 1(10), 40-51.

Zeichner, K. (2010). New epistemologies in teacher education. Rethinking the connections between campus courses and practical experiences in teacher education at the university. Interuniversity Journal of Teacher Education, 68(24.2), 123-150.

Zeichner, K. \& Noffke, S. (2001). Practitioner research. In V. Richardson (Ed.). Handbook of research on teaching. (4th ed.), 298-330. Washington DC: American Educational Research Association. 